\title{
Erratum: Strong interplay between stripe spin fluctuations, nematicity and superconductivity in FeSe
}

Qisi Wang, Yao Shen, Bingying Pan, Yiqing Hao, Mingwei Ma, Fang Zhou, P. Steffens, K. Schmalzl, T. R. Forrest, M. Abdel-Hafiez, Xiaojia Chen, D. A. Chareev, A. N. Vasiliev, P. Bourges, Y. Sidis, Huibo Cao and Jun Zhao

Nature Materials http://dx.doi.org/10.1038/nmat4492 (2015); published online 7 December 2015; corrected online 15 December 2015.

In the original version of this Letter published online, the $x$ axis of Fig. 2a was labelled incorrectly. In addition, tick labels have been added to the $x$ axes of Fig. $2 \mathrm{~b}$ and Fig. $2 \mathrm{~d}$. This has been corrected in all versions of the Letter. 